\title{
Comprehensive Personalized Information Access in an Educational Digital Library
}

\author{
Peter Brusilovsky \\ University of Pittsburgh \\ School of Information Sciences \\ Pittsburgh, PA 15260 \\ peterb@pitt.edu
}

\author{
Rosta Farzan \\ University of Pittsburgh \\ Intelligent Systems Program \\ Pittsburgh, PA 15260 \\ rosta@cs.pitt.edu
}

\author{
Jae-wook Ahn \\ University of Pittsburgh \\ School of Information Sciences \\ Pittsburgh, PA 15260 \\ jaa38+@pitt.edu
}

\begin{abstract}
This paper explores two ways to help students locate most relevant resources in educational digital libraries. One method gives a more comprehensive access to educational resources, through multiple pathways of information access, including browsing and information visualization. The second method is to access personalized information through social navigation support. This paper presents the details of the Knowledge Sea II system for comprehensive personalized access to educational resources and also presents the results of a classroom study. The study delivered a convincing argument for the importance of providing multiple information presentations modes, showing that only about $10 \%$ of all resource accesses were made through the traditional search interface. We have also collected some solid evidence in favor of the social navigation support.
\end{abstract}

\section{Categories and Subject Descriptors}

H.5.1 [Information Interfaces and Presentation]: Multimedia Information Systems - Hypertext navigation and maps.

\section{General Terms}

Documentation, Performance, Human Factors, Languages.

\section{Keywords}

Social navigation, information map, classroom study.

\section{INTRODUCTION}

The way computers are used in education is changing rapidly. Large repositories of educational material are being created, including educational digital libraries (EDL) such as the National Science Digital Library (NSDL) [17] and repositories of reusable learning objects $[11 ; 16]$. These repositories attempt to collect a large volume of high-quality material which has been carefully authored, reviewed and indexed with standard metadata. Teachers and students at all levels can greatly benefit from these

\footnotetext{
Permission to make digital or hard copies of all or part of this work for personal or classroom use is granted without fee provided that copies are not made or distributed for profit or commercial advantage and that copies bear this notice and the full citation on the first page. To copy otherwise, or republish, to post on servers or to redistribute to lists, requires prior specific permission and/or a fee.

JCDL'05, June 7-11, 2005, Denver, Colorado, USA

Copyright 2005 ACM 1-58113-876-8/05/0006 ...\$5.00.
}

repositories. A challenge for the researchers is to provide efficient tools and services which will allow the users of educational digital libraries to locate resources that are relevant to their individual goals, interests, and current knowledge.

Presently, educational digital libraries (EDL) rely on the traditional information retrieval approach as the only way to access educational resources in EDL. For example, NSDL focuses on providing powerful search and discovery services [10]. However, in any reasonably sized EDL it is very challenging to find the most appropriate resources among the hundreds of links returned by a search service. The results of several research projects show that the ability to choose appropriate links requires a relatively high level of background or domain knowledge [2; 15]. Even in relatively small EDL where a query typically returns dozens of hits, less prepared students may have problems narrowing it down to resources that are relevant. As the growth of EDL accelerates, search-only access may become practically unusable even for domain experts. A query that once yielded tens of hits now yields thousands [12].

This paper explores two ways to help students locate the most relevant resources in educational digital libraries. First, we attempt to provide more comprehensive access to educational resources in EDL. For the purpose of this paper we distinguish four major techniques of information access: ad-hoc information retrieval (IR), information filtering, hypertext browsing, and information visualization [5].

In ad-hoc information retrieval (IR), users get access to relevant information by issuing a query to an IR system or search engine and analyzing a ranked list of documents (for example, book records), which are returned as a result. In modern information filtering, a system actively recommends relevant documents to the users, on the basis of inferred or provided interest profiles. In hypertext browsing, a user attempts to find relevant documents by browsing links that interconnect documents within a collection. In information visualization, a set of documents is presented to the user using some visualization metaphor in 2 or 3 dimensions; the user observes or, in the case of interactive visualization, interacts with the visualized set to find the most relevant documents. While multiple research studies argue that different techniques of information access have different strengths that may complement each other, most EDL rely on IR as their sole method of information access. A few advanced projects such Walden's Paths [8] and Instructional Architect [14] attempt to supplement searchonly access with recommendations that are manually created by teachers through a path-building service or an advanced lesson- 
building service. We attempt to advance progress even further by providing all four ways of information access and then exploring the value of this comprehensive access.

Second, we attempt to help users locate resources that are relevant to their goals, knowledge, and interests through personalized access to resources [5]. Over the last two years we have been exploring a promising approach to personalization access known as social navigation [6]. Social navigation tries to solve the navigation problem by taking advantage of the natural human tendency to follow in the footsteps of other people with similar interests. We are trying to use this tendency toward social navigation to assist learners to find the right information easier and faster. We started exploring social navigation support in the context of our Knowledge Sea project, which is currently focused on helping beginning programming students find relevant readings among the hundreds of online tutorial pages that are distributed over the Web. Social navigation relies heavily upon user feedback from group members. User feedback can be gathered through explicit forms, such as direct ratings, or it may be measured in implicit formats, such as by counting visits to a page.

In our earlier studies of Knowledge Sea II we focused on information access through visualization and explored social navigation support based on implicit feedback collected through recording every time a learner navigates through the system [3]. Our results demonstrated that (1) information visualization is an efficient technique of information access within the EDL context and (2) social navigation support is highly appreciated by students. In our current work we attempt to use page annotations as an additional source of social feedback. This annotation feature enables students to write notes for each tutorial page and highlight different parts of the tutorial pages. In addition we supplemented the original visualization-centered Knowledge Sea system with other ways of information access in order to explore the value of a more comprehensive information access system. In the Fall Semester of 2004, the new version of the system, which had comprehensive techniques of information access and enhanced social navigation, was explored in a classroom study. The study showed that both comprehensive access and social navigation support are important for students using an EDL. This paper describes our approach, which offers social navigation support in a web-based environment for several techniques of information access (section 2) and then describes the evaluation of our approach through a classroom study (section 3 ). The last section of the paper illustrates some possible future directions for our research.

\section{KNOWLEDGE SEA}

Knowledge Sea is the platform we use to explore personalized information access. Currently, the system provides access to over 25,000 documents related to teaching $\mathrm{C}$ programming, distributed over the Web. The original goal of Knowledge Sea was to bridge the gap between open corpus online resources (such as online tutorials and textbooks) and closed corpus resources (such as lecture notes) through map-based horizontal navigation [4]. To help learners navigate from lecture handouts to relevant online tutorial pages, the Knowledge Sea system provided an information map. Every cell of the map included links to similar pages of open and closed corpus material, with adjacent cells presenting less similar material. While the information map is still the core of Knowledge Sea II, the system currently provides more comprehensive information access through the addition of information visualization, search, hypertext browsing, and direct recommendation. In addition, it employs social navigation support that is based on the learner's previous interaction with the system.

\subsection{Comprehensive Information Access}

An important feature of the new version of Knowledge Sea II (KSII) system is the support of several methods of information access. One of our goals is to assess the practical usage of each access method. Therefore in addition to traditional search service, Knowledge Sea supports map-based information access, hypertext browsing, and instructor recommendations. The following four types of information access are available in Knowledge Sea II (Figure 1):

1. Information visualization. Our traditional information map provides access to hundreds of pages grouped into cells by keyword-level similarity. Keywords and closed corpus landmark are provided to help students find the most relevant cells. When opened, each cell provides links to up to several dozen similar resources. In the evaluation section of the paper we refer to this access method as "Map".

2. Hypertext browsing. Typically, a C programming Web tutorial or textbook from KSII collection is organized as a tree of sections connected by links. KSII preserves these links and encourages learners to use them. Often students discover some relevant page by using map or search and then continue to explore the same tutorial along its own authored links. Alternatively, students can start browsing from the root page of each tutorial (root pages of all tutorials are listed on the front page of the system). While pages of some tutorials are not accessible through the map, every page in the system is accessible through browsing.

3. Search. Students are able to perform standard keyword-based search. Each search request returns a list of links to resources ordered by relevance to the query. KSII uses a standard, vectorbased search engine. The detail of the search engine is out of the scope of this paper.

4. Recommendation. Several tutorial pages are explicitly recommended by the instructor. Links to these pages are added to the list of resources for the corresponding lecture in the course portal. We expected that it will be the easiest way for students to access resources, since the portal is frequently used for a range of educational activities. We refer to this method of access as the "Instructor" method.

\subsection{Social Navigation Support}

Knowledge Sea II offers two kinds of social navigation support: traffic-based and annotation-based. As we mentioned above, traffic-based social navigation support was introduced in the first version of KSII [3] and annotation-based support was added in the current version. This section presents state of social navigation support in the most recent version of KSII that was used in our study. 


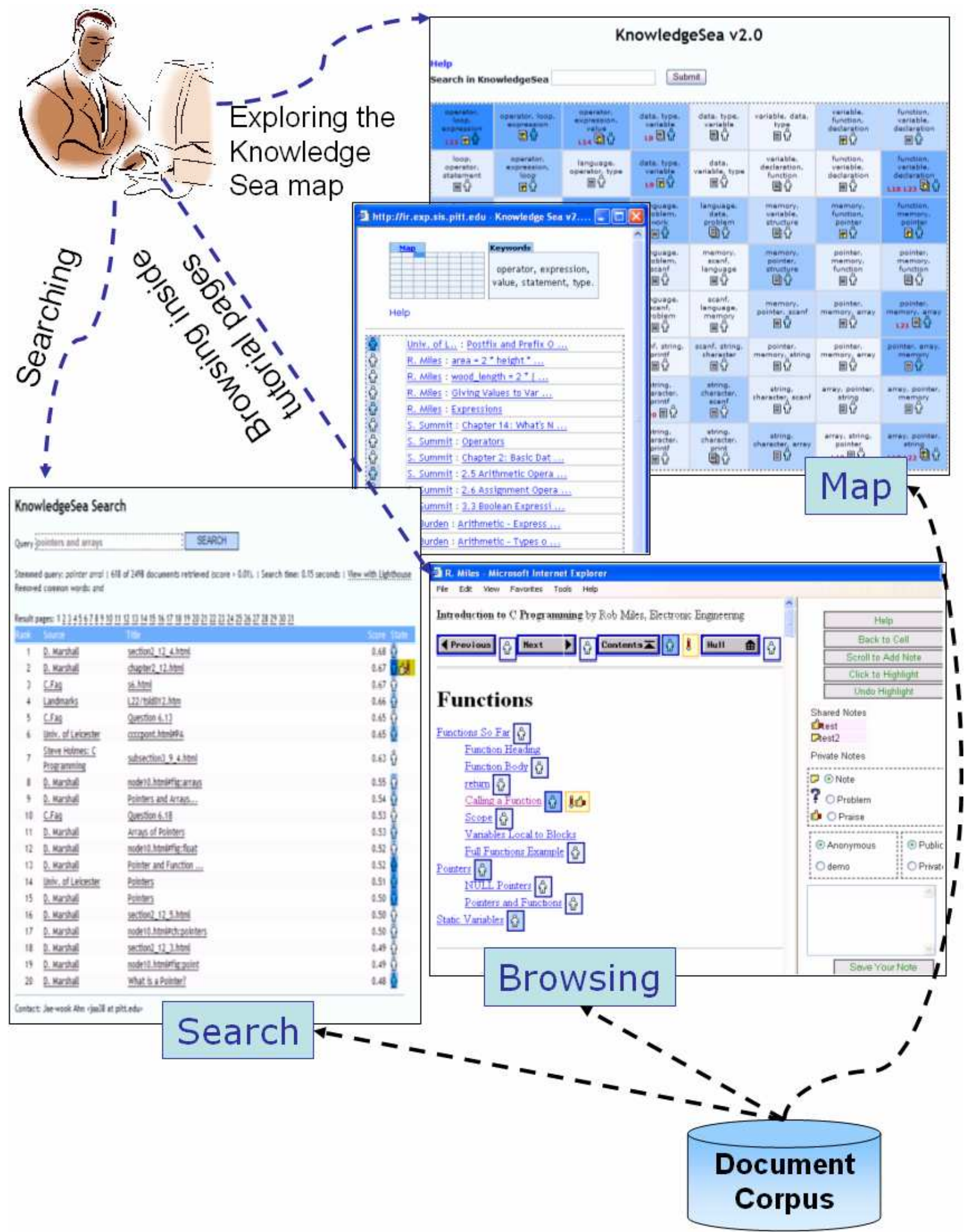

Figure 1. Overview of different information access method in Knowledge Sea: visualization, browsing, and search. 


\subsubsection{Traffic-based Social Navigation Support}

The idea of traffic-based social navigation support is to visualize the navigational history of each group of learners. The intensity of the background color of each cell on the map represents the intensity of group traffic [3]. The map starts with a very light shade of blue for each cell and, as the students progress, more frequently visited cells become darker and darker blue so that students can easily follow the footprints of others by visiting cells with darker backgrounds. Students can also view the history of their own interaction with the system. The color of the "human" icon shown in each cell reflects the number of cell visits done by the individual student. The more visits, the darker the color of the icon. In this way, students can compare their interaction with the system with those of their total community members by comparing the color of the human icon to the background color (Figure 2). This information helps the students to decide what cell to visit next.

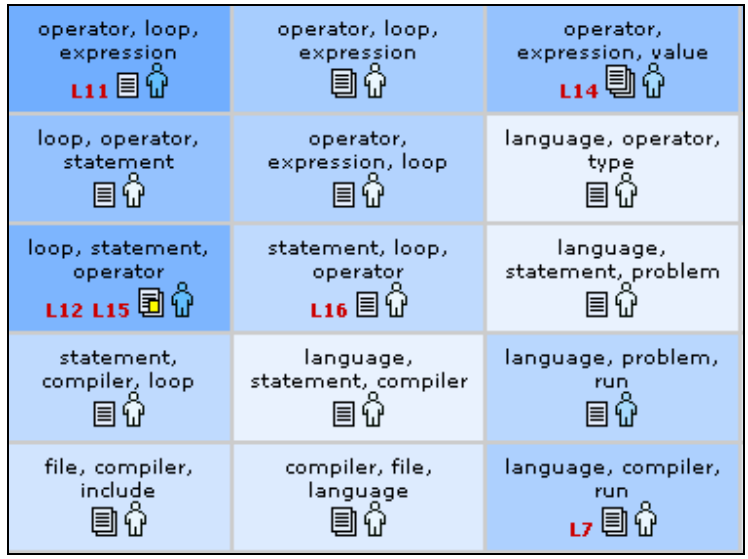

Figure 2. A fragment of the Knowledge Sea map.

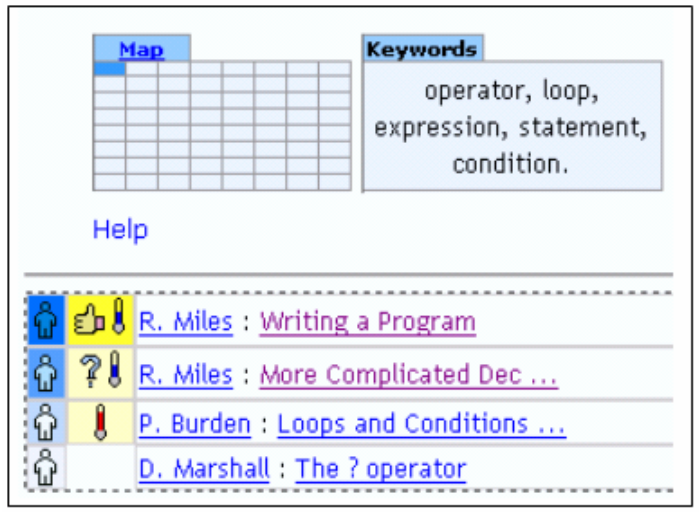

Figure 3. A fragment of a map cell with the list of resources.

When a student clicks on one of the cells of the map, the cell "opens" and shows the list of available resources inside the cell. Typically, behind each of these links is one section from a webbased C programming tutorial or book. Figure 3 presents a fragment of one the map cells. The top part of the cell shows a small map that presents the position of this cell in the Knowledge Sea map and the top five keywords of the open cell. The bottom part shows the list of resources. Each resource link has two parts: the identification of the tutorial it belongs to (typically, the author's name, which also serves as a shortcut to the root of the tutorial) and the title of the section.

A finer-grain traffic-based social navigation support is offered for each resource inside the cell. The left hand side column of the list of resources shows visual navigation cues that reflect individual and group traffic information. The same visualization approach is again being used here: the background color represents group traffic and the color of the human icon, individual traffic. The information in figure 3 shows that sections "Writing a Program" and "More Complicated Decisions" of the R. Miles textbook are quite popular among this group of students. We can also see that the student for whom these cues were generated has visited "Writing a Program" page more than the group and "More Complicated Decisions" less than the group. More details about traffic-based navigation support within the Knowledge Sea system can be found in [3].

The same traffic-based adaptive visual cues are consistently provided next to each resource link in KSII, in any context where this link may appear, except for the course portal. The browsing support component of the system dynamically processes all tutorial pages and adds corresponding traffic-based cues to the right of each link previously visited by the user of the group. For example, Figure 4 shows a fragment of a tutorial page from the R. Miles tutorial. All links on this page are created by the author and were dynamically enhanced with adaptive visual cues at runtime. Here we see, for example, the link to the same page "More Complicated Decisions" that was shown inside map cell on Figure 3 .

Finally, to keep it consistent, the KSII search engine generates the same traffic-based visual cues for each link when presenting results of each search (Figure 5). The links on the search results page are ordered by page relevance to the query. This relevance score is calculated in a traditional way for a vector model [9] and shown to the right of each link (as it is done by a number of search engines). The "social relevance" of links is expressed solely by social navigation cues and is not used to calculate this relevance. While existing content-based [13] and collaborative [7] adaptive search systems fuse the "raw" query relevance data with user or group relevance data into a combined rank, we decided to use two different approaches to show "raw" query relevance (ordering) and social relevance (visual cues). This is done for both - consistency and evaluation reasons. Indeed, keeping query relevance and group relevance separated allowed us to compare the value of both mechanisms in this study.

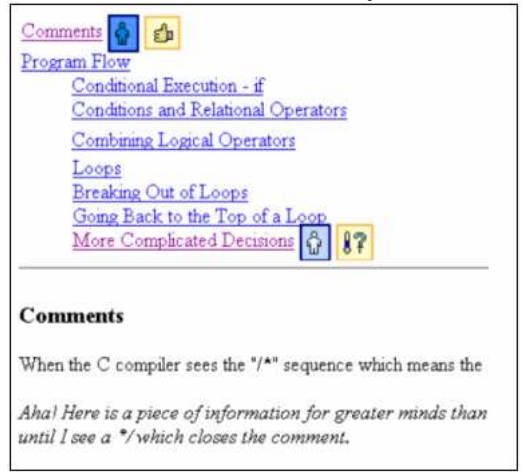

Figure 4. Links inside tutorial pages annotated with social navigation support 


\subsubsection{Annotation-based Social Navigation Support}

Annotation-based social navigation support is the newest feature of Knowledge Sea that has been pioneered in the last version of KSII. Based on student feedback collected during the evaluation of the second version of the system, we attempted to provide a more reliable form of social navigation support that would supplement simple traffic information. Annotation-based support harnesses the power of page highlighting and annotation made by students. An important feature of the Knowledge Sea system is the ability to annotate tutorial pages. Students are able to write notes for each page and highlight different sections of the page while reading each tutorial page. In addition to the personal benefits of annotating while reading, which include helping the learners to remember, clarify, and make sense of the material while trying to reshape the information [1]; presence of annotation creates an important trail for other learners to follow. AnnotatED, the annotation component of Knowledge Sea, provides a rich set of features to support annotating activity. Students can specify the usefulness of the marked page by associating a positive, negative or general type to their notes. Also they can specify whether or not they want to share the notes with others and also if they want to write the note anonymously or with their username. All this information assists the author of the annotation and other learners when they later consider access to the annotated material.

As can be seen in Figures 3, 4, and 5, some of the links are enhanced with more than traffic information. The additional icons are designed to support learners with more emphatic information, based on annotations provided by this student and the group of students. The "question mark" and "thumbs up" icons correspond to a negative and a positive comment to this page made by the current student. If a student writes a positive note for a page, the link to that page will be augmented with a "thumbs up" icon in any context where this link is shown. If a student writes a negative annotation for a page, the page will be annotated with a "question mark" icon. If a student has written a neutral note for a page, the icon will show a sticky note. In this way the student can easily locate previously annotated pages. In particular, it helps the student to navigate back to pages that were previously found useful and to ignore pages that were not considered to be beneficial.

In addition, some of the links are annotated with a thermometer icon. The thermometer icon presents the overall "annotation temperature" of the page showing whether the page has received more positive annotation or more negative annotation from the group. If the thermometer shows a positive temperature, the page has more positive annotations. If it shows negative (blue) temperature, the page has overall received more negative annotations. Therefore, the thermometer icon assists students in deciding which pages to visit by recommending pages with positive temperature and warning about pages with negative temperature. For example, in Figure 3, the link to "R. Miles: Writing a Program" is annotated with both a thumbs up icon and a thermometer showing a low temperature. This means the current student has found the page useful, but overall the page has received more negative annotation than positive annotation. At the same time, the page "Loops and Conditions," which has not been visited by the current student, has a high-positive annotation temperature.
Similar to traffic-based visual cues, annotation-based cues also apply the color-intensity paradigm to express information about the density of annotations made by the group or the user. The intensity of the yellow background color represents the density of group annotation and the intensity of the foreground icon color (thumbs up, question mark, or sticky note) represents the density of the individual annotations.

\begin{tabular}{|c|c|c|c|}
\hline \multicolumn{4}{|c|}{ KnowledgeSea Search } \\
\hline \multicolumn{3}{|c|}{ Query array pointer string } & SEARCH \\
\hline \multicolumn{4}{|c|}{ Stemmed query: arral pointer string 1812 of 2498 documents retrieved iscore $=0.01$} \\
\hline \multicolumn{4}{|c|}{ Result pages: 123456789 to 11 12 131415 16 $171819202122 \geq 32425262728$} \\
\hline Pank & Soures & Titis & Sugre stote \\
\hline 1 & Landmarks & $\underline{122 / \text { tsidot2.htm }}$ & 0.728 \\
\hline 2 & $\frac{\text { Steve Holmes: } C}{\text { Programming }}$ & subsection 3.9 .4$, html & 0.60 \& \\
\hline 3 & s. Summit & 10.7 strings & $0.58 \%$ \\
\hline 4 & D. Marshall & section2_12_4.htm! & $0.58 \mathrm{G}$ \\
\hline 5 & D. Marshall & chapter2_12.html & 0.55 E. Bd \\
\hline 6 & C.Fag & $\underline{s 6 . h t m l}$ & $0.55 \%$ \\
\hline 7 & C.Fag & Question 6.13 & 0.538 \\
\hline 8 & Univ. of Leicester & ccccpont.htmixpA & 0.538 \\
\hline 9 & Univ. of Leficester & cocestrghtmitssa & $0.50 \%$ \\
\hline 10 & P. Burden & chap6.intro.html & 0.49 है \\
\hline 11 & Univ. of Leicester & Strings & $0.48 \%$ \\
\hline 12 & D. Marshall & node10 htminfig:arrays & 0.48 \& \\
\hline 13 & C.Fag & Question 8.2 & 0.47 Q \\
\hline 14 & D. Marshall & Pointers and Arrays... & 0.47 हि \\
\hline 15 & D. Marshall & node10, htminfig:float & 0.45 षి \\
\hline 16 & D. Marshall & Arrays of Pointers & 0.45 .8 \\
\hline 17 & D. Marshall & Pointer and Function .... & $0.45 \mathrm{f}$ \\
\hline 18 & D. Marshall & section2_12_3.hitml & $0.45 \overline{8}$ \\
\hline 19 & C.Fag & Question 6.18 & 0.44 ही \\
\hline 20 & D. Marshall & Pointers & 0.43 . \\
\hline
\end{tabular}

Figure 5. The search interface

\section{EVALUATION}

The new version of Knowledge Sea II with enhanced social navigation and comprehensive information access was evaluated in a classroom study during the Fall 2004 semester. The focus of the study was the evaluation of social navigation support in its assistance to students looking for important information. In addition, we attempted to evaluate the importance of providing multiple techniques of information access to the learners. The system was made available to students of an introductory programming course at the University of Pittsburgh. At the end of the course, the students who used the system were asked to fill in an anonymous questionnaire. The use of the system and the questionnaire were not mandatory. Among 15 students enrolled, 13 used the Knowledge Sea system and 10 responded to the questionnaire. This section presents the results of the students' response analysis and the analysis of information access logs.

\subsection{Usefulness of Providing Multiple Methods to Access Information}

To evaluate the importance of presenting multiple methods of information access, we compared the frequency of student access to different groups of resources - categorizing their location and access type. Since the presence of a resource on the map and in 
the list of links recommended by the instructor can seriously influence the resource access profile, we attempted to calculate the access profile separately for four main groups of links. Table 1 shows the number of resources in each of the categories. As we see, among over 25,000 resources in the system, 894 are accessible through the map and the rest, only by search and browsing. Among 15 resources directly recommended by the instructor, 13 are also available through the map. During the semester we registered 825 accesses to 377 resources. The access distribution over the four resource categories is shown in Table 2. To compare the access rate to pages in different groups we calculated the normalized access rate for each group by dividing the number of accesses by the total number of pages in the group. This parameter can be considered as the probability of accessing each kind of page.

As the data shows, the documents directly recommended by the instructor through the course portal have the highest access rate. Given the small number of recommended documents and a oneclick access to them, this is the result we expected. Less expected is the large effect of the information map. While all documents can be equally accessed by browsing and search, documents that are not present on the map have a dramatically smaller chance to be accessed. This difference is very significant ( $p$-value $=0.0001$ ). Even when the document is provided in the instructor's list, the map plays a role in increasing the chance of a visit (5.5 versus $3.5)$.

Table 1. Number of documents

\begin{tabular}{l|r|r|r} 
& On Map & \multicolumn{1}{|c|}{ Not On Map } & \multicolumn{2}{|l}{ Total } \\
\hline On List & 13 & 2 & 15 \\
\hline Not on List & 881 & 24,553 & 25,434 \\
\hline Total & 894 & 24,555 & 25,449
\end{tabular}

Table 2. Number of accesses

\begin{tabular}{l|r|r|r} 
& \multicolumn{1}{|c|}{ On Map } & \multicolumn{1}{|c|}{ Not On Map } & \multicolumn{2}{|c}{ Total } \\
\hline On List & 71 & 7 & 78 \\
\hline Not on List & 401 & 346 & 747 \\
\hline Total & 472 & 353 & 825
\end{tabular}

Table 3. Normalized access rate for different methods

\begin{tabular}{l|r|r|r} 
& On Map & \multicolumn{1}{|c}{ Not On Map } & \multicolumn{2}{|c}{ Total } \\
\hline On List & 5.46 & 3.5 & 5.2 \\
\hline Not on List & 0.46 & 0.014 & 0.03 \\
\hline Total & 0.53 & 0.014 & 0.03
\end{tabular}

As noted above, the profile of information access was calculated separately for the following three categories of documents (Figure $6)$.

1. Category 1: Documents in the list recommended by the instructor. In this category we united all 15 recommended pages, regardless of their presence on the map. 13 of these pages were accessible through all four ways of information access. As it can be seen in the first column of Figure 6, only about $55 \%$ of the access to these pages is done through "Instructor" method. The "Browsing" and "Map" method each accounted for about $20 \%$ of the accesses and "Search" for just $5 \%$ of the accesses. It shows the value of the browsing and map method, even for documents that were one click away from the course portal.

2. Category 2: Documents accessible from the map. This category includes all documents accessible through the map, search, and browsing, but not through recommended links. Among 377 accessed pages, 193 pages belonged to this category. As shown in the second column of Figure 6 about $60 \%$ of accesses to documents of this category were done through the map, about $30 \%$ through browsing, and, surprisingly, just $10 \%$ through search. As we expected, the organized map is the easiest method for the students to access information. In our system the power of the map was enhanced since it is easy to to follow the footprints of others by visiting cells with darker backgrounds and student annotations.

3. Category 3: Documents not accessible from the map. This category includes 24,555 documents that were accessible only by search and browsing. Since search is the main access methods in digital libraries, we expected that most of the pages in this category would be accessed by search. However, surprisingly, only $10 \%$ of the accesses were done through search and the rest were done through browsing.

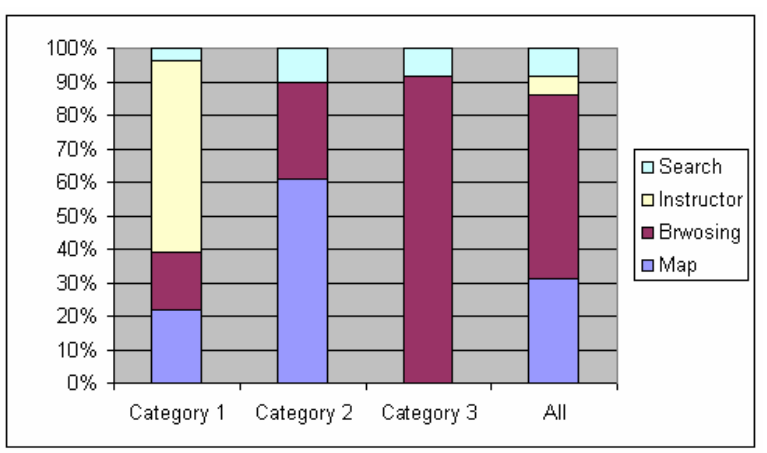

Figure 6 - Comparison of different accessing methods

We have also looked at the top 20 accessed documents and checked the most frequently used access methods for these documents. Overall, the top 20 accessed documents were accessed 214 times. As shown in Table 4, again the map method generated the highest percentage of accesses, followed closely by the browsing method. This shows that finding information through the socially annotated map and browsing are the two favorite ways for the students to navigate, even surpassing the explicit recommendations of the instructor.

Table 4. Accessing method for the top 20 accessed pages

\begin{tabular}{|l|l|l|l|}
\hline Map & Browsing & Instructor & Search \\
\hline $44 \%$ & $35 \%$ & $12 \%$ & $10 \%$ \\
\hline
\end{tabular}

The least expected result of the study was the relatively low value of search-based access. We have added search-based access in the third version of the system, expecting that the ability to search 
would at least double the use of resources. While the students appreciated the search-based access, only about $10 \%$ of accesses were done through search, making it the least useful method of information access for our students. The low value of search can be also be observed by counting the number of students who used search at least once while working with Knowledge Sea. Out of 13 students who used Knowledge Sea during the semester, only 7 students used search at all. We think that our data clearly shows how critical it is to provide different methods of information access in digital libraries, in order to complement the traditional search-based access.

\subsection{The Usefulness of Social Navigation Support: Questionnaire Answers}

To evaluate the usefulness of social navigation support, we looked at student responses to the survey questions regarding social navigation support.

One set of questions asked the students to agree or disagree with statements about the usefulness of the following features related to annotation-based social navigation support:

1. Density of public annotations made by the total group of students is represented by darkness of the background color

2. Temperature of the tutorial pages represents the overall number of positive and negative annotations, and is presented in the thermometer icon.

3. Individual evaluations in the form of specific tutorial page annotations is represented by thumbs up, question mark or sticky note icons.

As can be seen in the first three columns of Figure 7, in all cases more than $70 \%$ agree or strongly agree that these features were useful. The champion is the annotation temperature: all students agreed that the presence of the thermometer helps them to decide what page to visit next.

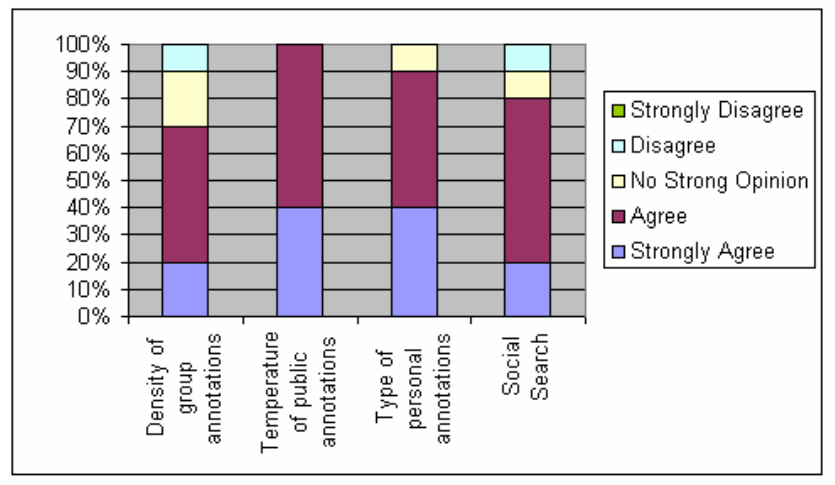

Figure 7 - Students' attitude towards annotation based social navigation

We also asked students about the usefulness of adding social navigation support into the search result. The last column in Figure 7 shows that $80 \%$ of students found useful the addition of social navigation support to search results.

Another set of questions asked students about specific ways to display group traffic and user traffic on the Knowledge Sea map and inside pages and cells. As presented in Figure 8, at least $70 \%$ of the students agreed or strongly agreed that the current way of presenting traffic-based social navigation on both the map and inside pages is useful and appropriate. The highest agreement was on the presentation of group traffic on the Knowledge Sea map $(90 \%)$ - probably because different shades of blue are more easy to distinguish on the map, thus helping students to choose the next cell they wish to visit on the map.

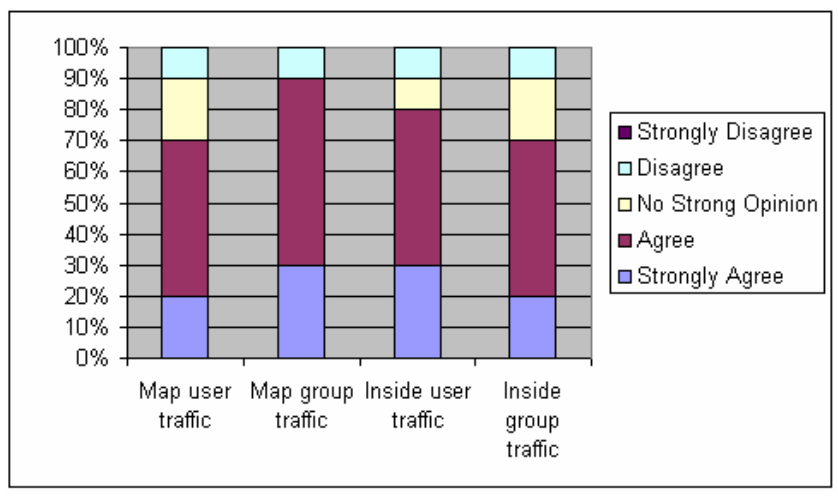

Figure 8. Student attitudes toward the presentation of traffic-based social navigation

\subsection{The Usefulness of Social Navigation Support: Log Analysis}

\subsubsection{Does Social Navigation Support Lead Students to "Good" Pages?}

Using system logs, we analyzed the correlation of the importance of the pages with our traffic-based navigation support. Our gold standard for rating the importance of pages is by what student find good and important. Therefore, we look for pages with positive annotations made by students. To start with, annotations tagged by students as praise are considered positive annotations. In addition, looking into our data, we have observed that students often generally write a lot of very positive annotation (Out of 45 general notes written over 2 semesters, 26 can be considered as positive annotations). Therefore, we consider annotations tagged by student as general but positive in content to be positive annotations as well. To assess our traffic-based navigation support, we looked at the navigation behavior of the students over the "good and important" pages.

Out of over 25000 documents available in the Knowledge Sea system 377 documents have been accessed by the students during the study. Out of 377 documents, 38 documents have been annotated by last semester's students while another 36 have been annotated over previous semesters the system was used. Therefore, a total of 74 documents out of 377 have been annotated by students. Out of these 74, 36 documents have positive annotations and positive temperature. Figure 9 shows the normalized access rate for pages with and without annotation as well as pages with positive annotations. As can be seen in the figure, "good and important" pages are accessed twice as often. Thus page traffic average is a good indicator of page quality. "Good and important" pages will have a generally darker background, according to our traffic-based social navigation support algorithm. It is interesting to note that the number of clicks on pages with positive annotations is not higher than pages with any other kind of annotation, which means that pages with 
positive annotations would not necessarily have darker backgrounds.

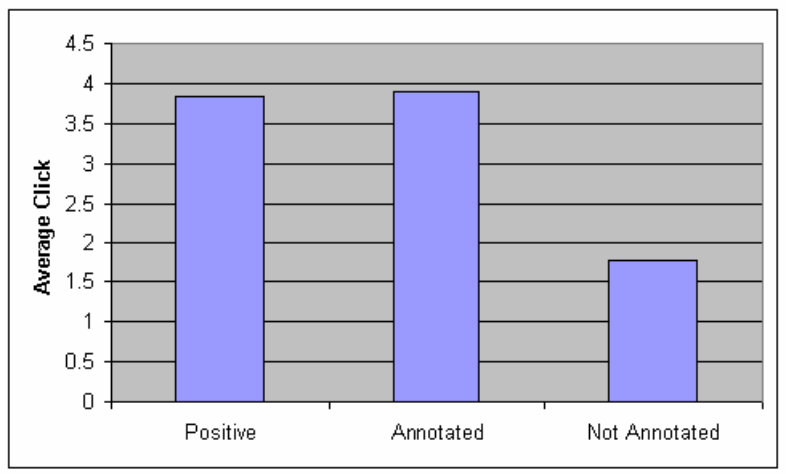

Figure 9. Average click number over pages with different annotation status

\subsubsection{Do Students Take Social Navigation Cues into} Account When Selecting Resources?

Social navigation cues could lead students to good pages. However, do the students ever notice these cue and take them into account when selecting tutorial pages? To answer this question, we looked at the effect of the presence of public annotation on the page-visiting behavior of students. We compared the percentage of page accesses done before and after the first public annotation was made on each page. To have a more accurate result, the percentage of activity was normalized over the number of possible days for activity. As can be seen in Figure 10, in most cases, more than $50 \%$ of accesses were made after the page was first marked with a public annotation. Out of 34 pages with public annotations, 28 pages have been visited more after the first public annotation was made. As we expected, this result suggests that public annotation attracts the attention of students and influences their decision about which page they should visit next.

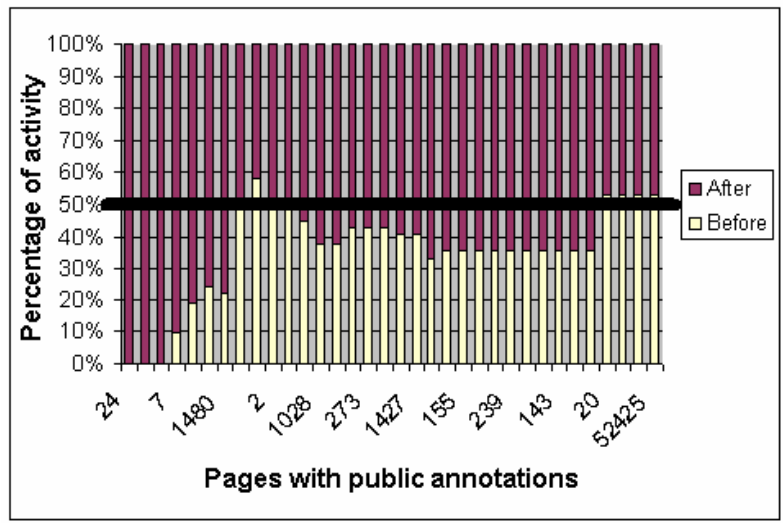

Figure 10. Percentage of activity before and after the first public annotation, for pages with public annotations

For better assessment of effect of social navigation on navigation behavior, we compared the normalized access rate for pages with different statuses. The status of a page includes information about the number of clicks before the current access, the number of public annotations made before the current access, and the temperature of annotations before the current access. Figure 11 shows that social navigation support, which is related to page status, has an important effect on students' navigational choices. As mentioned above, the normalized access rate is computed by dividing the number of accesses to pages in this category by the total number of available pages in this category. Table 5 shows how the normalized access rate is computed for each status. As the data shows, the chance of visiting an arbitrary resource in $\mathrm{KSII}$ is very low (close to 0 ) since there is a large number of resources in the system. Social navigation support and specifically annotation-based social navigation increases the chance of accessing a resource dramatically.

Table 5. Comparing navigation for pages with different statuses

\begin{tabular}{|l|c|c|c|c|}
\hline & $\begin{array}{l}\text { No } \\
\text { Group } \\
\text { Traffic }\end{array}$ & $\begin{array}{l}\text { Group } \\
\text { Traffic }\end{array}$ & Annotation & $\begin{array}{l}\text { Positive } \\
\text { Temperature }\end{array}$ \\
\hline \# of pages & 24,748 & 377 & 38 & 18 \\
\hline \# of Clicks & 282 & 465 & 118 & 43 \\
\hline Access rate & 0.011 & 1.23 & 3.1 & 2.39 \\
\hline
\end{tabular}

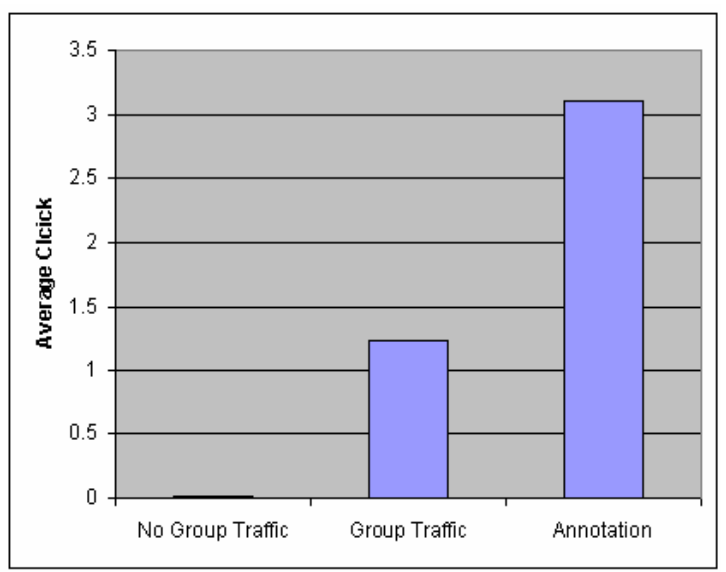

Figure 11. Comparing average click for pages with different statuses

\subsubsection{Does It Make Sense to Annotate Search Result with Social Navigation Cues?}

We have also assessed the effect of social navigation support on how the search results are used. We expected that students will prefer pages with visible group traffic and public annotations. We also hoped that the students may prefer socially annotated pages even if they are located relatively low on the results page. As shown in

Table 6, the number of pages visited from links within the search result is, indeed, higher when these links have icons showing group traffic than when no group traffic is represented. On the other hand, the average rank (position in the list of results) of pages with group traffic is lower than pages without group traffic, which means that selected pages with group traffic were located higher in the list than selected pages with no traffic. For selected pages with "strong" social navigation cues such a high traffic (indicated by dark blue) or annotations the average rank is even lower (Tables 6 and 7). This result shows that students preferred 
pages that have two evidences of quality - lower rank (higher relevance score) and presence of social navigation cues.

Table 6. Effect of traffic-based social navigation on visiting pages within search results

\begin{tabular}{|l|l|l|l|}
\hline & $\begin{array}{l}\text { No group } \\
\text { traffic }\end{array}$ & $\begin{array}{l}\text { Any group } \\
\text { traffic }\end{array}$ & $\begin{array}{l}\text { High group } \\
\text { traffic only }\end{array}$ \\
\hline Average rank & 8.54 & 6.48 & 5.94 \\
\hline Selection count & 24 & 29 & 17 \\
\hline
\end{tabular}

Table 7. Effect of annotation-based social navigation on visiting pages within search results

\begin{tabular}{|l|l|l|}
\hline & $\begin{array}{l}\text { Without public } \\
\text { annotation }\end{array}$ & $\begin{array}{l}\text { With public } \\
\text { annotation }\end{array}$ \\
\hline Average Rank & 7.55 & 5.75 \\
\hline Selection Count & 49 & 4 \\
\hline
\end{tabular}

Since the "simple" approach to determine the value of social navigation cues in the search interface provided little insight, we had to use a more advanced approach. We decided to compare effective and random relative access rate for links with low rank (on the top of the list) and links with traffic-based cues. The random relative access rate tells which fraction of clicks will be made on links with a specific property if the user selects links in the search results list randomly. Basically, it shows how often the links with this property appear in the search results list. The effective relative access rate reports the real fraction of links with the target quality among all accessed links. If the effective relative access rate is higher than random, it means that the links with this quality encourage user to access them. If the effective relative access rate is lower than random, it means that the links with this quality discourage user from accessing them.

The first question to answer is "do students prefer links with low rank" (we consider top three documents in the search results list with rank 1 to 3 as low ranked). Since every result page shows 20 links, the random relative access rate for top three ranked documents is $3 / 20=0.15$. Effectively, students accessed 53 documents from different search results lists and out of these 53, 16 were among the top 3 documents. Therefore the effective relative access rate is $16 / 53=0.3$, which is twice the random $(0.15)$. This is evidence that the students do take the document rank into account preferring links on the top of the list (one may say that this is a rather obvious result).

The second question to answer is "do students prefer links with traffic-based social navigation cues". Answering this question we attempted to evaluate separately links with any visible past traffic (number of past clicks $>1$ ) and links with higher traffic (number of past clicks $>2$ ). The reason is that the links with one past click were annotated with a very light blue color, which, we afraid, some users might ignore. The color for links with two clicks was darker and clearly visible. The links with 3 and more past clicks were annotated with reasonably dark blue color and were hard to ignore.
Computing random relative access rate for links with group traffic was a complicated procedure. For each of the 53 cases of link access we had to re-create the group traffic accumulated at the time of access to understand how many links with social cues the user saw when making the selection. For each case we calculated the random relative access rate by dividing the number of links with target level of traffic to the total number of links. Then we averaged the probabilities over all 53 cases and found that for pages with visible traffic the random relative access rate is equal to 0.08 (Table 8). Out of 53 cases, students choose 17 documents from the visible traffic category. Therefore the effective relative access rate for links with visible traffic is $17 / 53=0.32$, which is four times higher than random access (0.08). Similar ratio (0.05 to 0.19 ) was obtained for links with high traffic. This result shows that students do prefer links with visible group traffic. Moreover, the ratio of their effective access rate to random is twice as higher for pages with visible traffic than for pages in the top of the results list. It provides evidence that pages with visible group traffic are more attractive to students than top pages returned by the search engine.

Table 8 - Effect of annotation-based social navigation on visiting pages within search results

\begin{tabular}{|l|l|l|}
\hline & Random Chance & Access Rate \\
\hline Group Traffic $>1$ & 0.08 & 0.32 \\
\hline Group Traffic $>2$ & 0.05 & 0.19 \\
\hline
\end{tabular}

\section{CONCLUSIONS and FUTURE WORK}

In our Knowledge Sea II system, we have attempted to help students access resources that are the most relevant and useful to them, by providing multiple techniques of information access and enhancing these techniques with social navigation support. We think that our study provides a convincing argument for the importance of presenting multiple methods of information access in educational digital libraries. Only about $10 \%$ of all resource accesses were made through the traditional search interface. At the same time, map-based access and hypertext browsing accounted for the majority of accesses. In particular, map-based navigation, which generated $44 \%$ of our student's accesses, should be considered an important addition to any EDL.

In addition, the study of Knowledge Sea presented some promising results in favor of social navigation support. We have observed that important pages receive larger number of clicks and thus by providing traffic-based navigation support, we can draw students' attention toward important pages. We have also observed that the presence of public annotation does influence students' navigation behavior and assists them in deciding which page to visit next.

To obtain more reliable data about the value of social navigation support, we need to explore this technique with a dramatically larger number of users. One of our plans is to explore social navigation support in the context of some broadly used EDL that contains a larger number of resources and a diverse community of users. We are very interested in collaborating with developers of EDL who would also be eager to explore the addition of more comprehensive personalized information access to their resources. 


\section{REFERENCES}

[1] Bonifazi, F., Levialdi, S., Rizzo, P., and Trinchese, R. UCA: A web-based annotation tool supporting e-learning. In: Proc. of Working Conference on Advanced Visual Interfaces: AVI 2002, ACM Press, 123-128.

[2] Brusilovsky, P. Adaptive navigation support in educational hypermedia: the role of student knowledge level and the case for meta-adaptation. British Journal of Educational Technology, 34, 4 (2003), 487-497.

[3] Brusilovsky, P., Chavan, G., and Farzan, R. Social adaptive navigation support for open corpus electronic textbooks. In: De Bra, P. and Nejdl, W. (eds.) Proc. of Third International Conference on Adaptive Hypermedia and Adaptive WebBased Systems (AH'2004), (Eindhoven, the Netherlands, August 23-26, 2004), 24-33.

[4] Brusilovsky, P. and Rizzo, R. Using maps and landmarks for navigation between closed and open corpus hyperspace in Web-based education. The New Review of Hypermedia and Multimedia, 9 (2002), 59-82.

[5] Brusilovsky, P. and Tasso, C. Preface to special issue on user modeling for Web information retrieval. User Modeling and User Adapted Interaction, 14, 2-3 (2004), 147-157.

[6] Dieberger, A., Dourish, P., Höök, K., Resnick, P., and Wexelblat, A. Social navigation: Techniques for building more usable systems. interactions, 7, 6 (2000), 36-45.

[7] Freyne, J. and Smyth, B. An experiment in social search. In: De Bra, P. and Nejdl, W. (eds.) Lecture Notes in Computer Science, Vol. 3137. Springer-Verlag, Berlin, 2004, 95-103.

[8] Furuta, R., III, F.M.S., Francisco-Revilla, L., Hsieh, H., Karadkar, U., and Hu, S.-C. Ephemeral Paths on the WWW: The Walden's Paths Lightweight Path Mechanism. In: Bra, P.D. and Leggett, J. (eds.) Proc. of WebNet'99, World Conference of the WWW and Internet, (Honolulu, HI, Oct. 24-30, 1999), AACE, 409-414.
[9] Korfhage, R.R. Information storage and retrieval, Wiley Computer Publishing, N.Y., 1997.

[10] Lagoze, C. and al., e. Core services in the architecture of the national science digital library (NSDL). In: Marchionini, G. and Hersh, W. (eds.) Proc. of ACM/IEEE-CS Joint Conference on Digital Libraries, (Portland, OR, July 14-18, 2002), ACM Press, 201-209.

[11] Laleuf, J.R. and Spalter, A.M. A component repository for learning objects: A progress report. In: Proc. of The first ACM/IEEE-CS joint conference on Digital libraries, (Roanoke, Virginia, United States, 33-40.

[12] Larsen, R.L. Relaxing Assumptions ... Stretching the Vision: A Modest View of Some Technical Issues. D-Lib Magazine, 3, April (1997), available online at http://www.dlib.org/dlib/april97/04larsen.html.

[13] Micarelli, A. and Sciarrone, F. Anatomy and empirical evaluation of an adaptive Web-based information filtering system. User Modeling and User Adapted Interaction, 14, 159-200 (2004).

[14] Recker, M. Instructional Architect, Logan, UT, Utah State University, 2004, available online at http://ia.usu.edu/

[15] Specht, M. and Kobsa, A. Interaction of domain expertise and interface design in adaptive educational hypermedia. Computer Science Report, Eindhoven University of Technology, Eindhoven (1999) 89-93

[16] Verhoeven, B., Cardinaels, K., Van Durm, R., Duval, E., and Olivié, H. Experiences with the ARIADNE pedagogical document repository. In: Proc. of ED-MEDIA'2001 - World Conference on Educational Multimedia, Hypermedia and Telecommunications, (Tampere, Finland, June 25-30, 2001), AACE, 1949-1954.

[17] Zia, L.L. The NSF National Science, Technology, Engineering, and Mathematics Education Digital Library (NSDL) Program: New Projects in Fiscal Year 2003. D-Lib Magazine, 10, 3 (2004). 